\title{
Distortions of Experimental Muon Arrival Time Distributions of Extensive Air Showers by the Observation Conditions
}

\author{
R. Haeusler ${ }^{a}$ A.F. Badea ${ }^{b, c}$ H. Rebel ${ }^{a, d, 1}$ I.M. Brancus ${ }^{c}$ \\ J. Oehlschläger ${ }^{a}$ \\ ${ }^{a}$ Forschungszentrum Karlsruhe, Institut für Kernphysik, 76021 Karlsruhe, \\ Germany. \\ ${ }^{\mathrm{b}}$ University of Karlsruhe, Institut für Experimentelle Kernphysik, 76021 \\ Karlsruhe, Germany. \\ ${ }^{\mathrm{c}}$ National Institute of Physics and Nuclear Engineering, 76900 Bucharest, \\ Romania. \\ ${ }^{\mathrm{d}}$ University of Heidelberg, Faculty of Physics and Astronomy, 69120 Heidelberg, \\ Germany.
}

\begin{abstract}
Event-by-event measured arrival time distributions of Extensive Air Shower (EAS) muons are affected and distorted by various interrelated effects which originate from the time resolution of the timing detectors, from fluctuations of the reference time and the number (multiplicity) of detected muons spanning the arrival time distribution of the individual EAS events. The origin of these effects is discussed, and different correction procedures, which involve detailed simulations, are proposed and illustrated. The discussed distortions are relevant for relatively small observation distances $\left(R_{\mu}<200 \mathrm{~m}\right)$ from the EAS core. Their significance decreases with increasing observation distance and increasing primary energies. Local arrival time distributions which refer to the observed arrival time of the first local muon prove to be less sensitive to the mass of the primary. This feature points to the necessity of arrival time measurements with additional information on the curvature of the EAS disk.
\end{abstract}

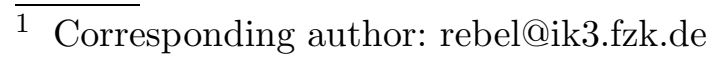

Preprint submitted to Elsevier Preprint

9 November 2018 


\section{Introduction}

The pioneering studies of the EAS disk structure in the early 1950's by Bassi, Clark and Rossi [1] have been followed by increasing efforts (e.g. [2-8]) to detail the information, up to recent measurements with modern detector arrays [9-11] like KASCADE [12]. Especially for the EAS muon component [12-14] the basic interest arises from the question, how well does the temporal EAS structure reflects the longitudinal profile and how well do muon arrival time distributions map the muon production heights, in particular when observed at sufficiently large distances from the shower axis, where the path-length effects dominate [16]. Via these features muon arrival time distributions should carry some information on the mass of the EAS primaries, unless the intrinsic fluctuations of the muon generation processes and limitations of the detector response do obscure the discrimination. Experimental studies with these aspects are a current subject of the KASCADE experiment [17-20], taking use of the timing and muon detection facilities of the KASCADE Central Detector $[21,22]$. Experimental muon arrival time distributions have experienced various intriguing distortions which depend not only on the time resolution of the timing detectors, but also on the multiplicity, i.e. the number $n$ of registered muons spanning the arrival time distribution of the single EAS event. Thus the shapes of the muon arrival time distribution are affected by the efficiency of

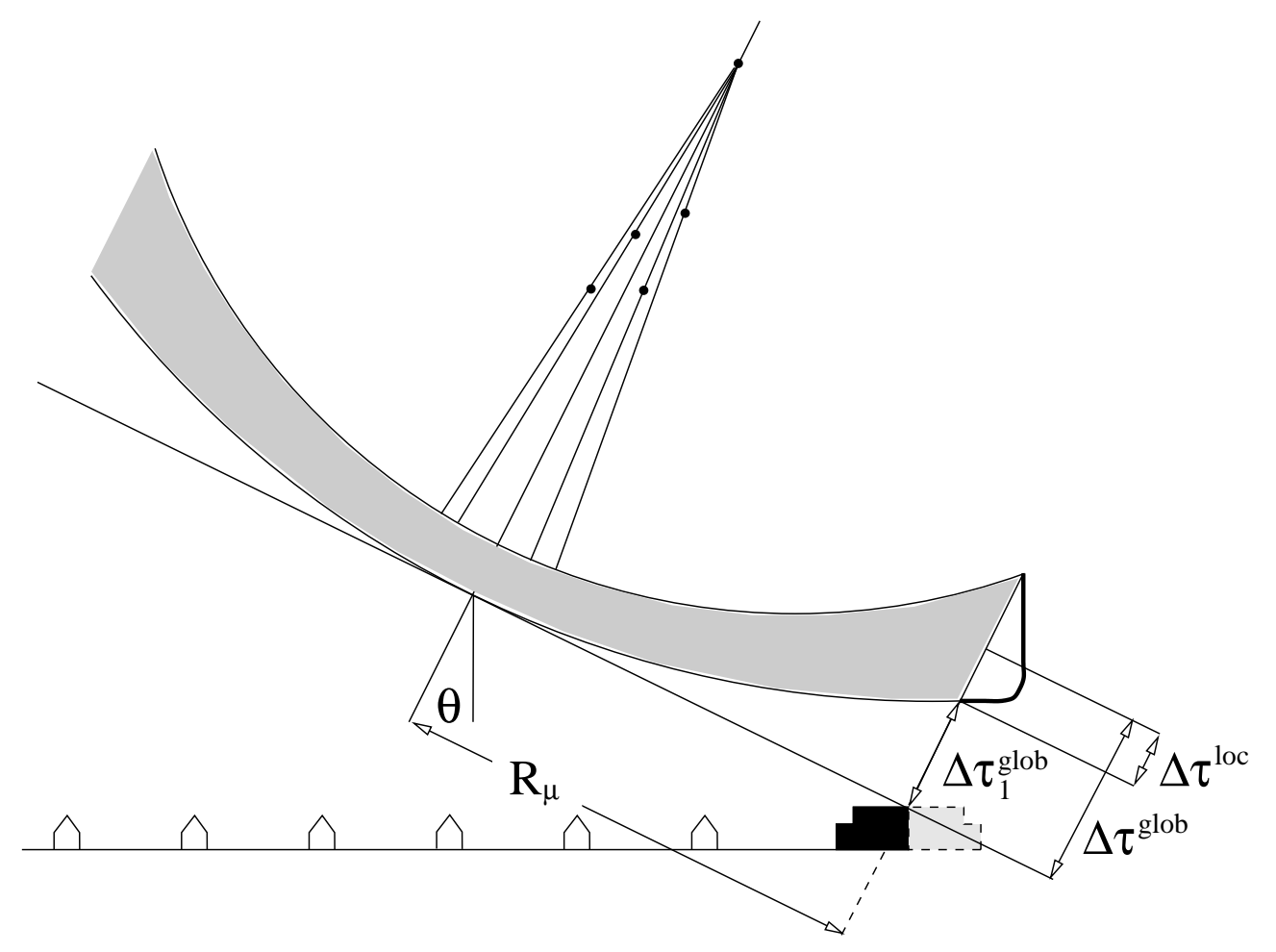

Fig. 1. Characterisation of the EAS temporal structure by global and local arrival time parameters. 
the detection system registering the fluctuating number of EAS muons. In this note we briefly discuss these multiplicity effects entangled with the response and the time resolution of the apparatus, and we suggest some procedures to reveal the basic time structure of the observed EAS. Fig. 1 indicates the used terminology (see also [17]). Arrival times of muons, registered by the timing detectors at a certain distance $R_{\mu}$ from the shower axis have to refer to a defined zero time. For the reference zero the arrival time $\tau_{c}$ of the shower core in the detection plane could be used (global arrival times). The correction $\pm R_{\mu} \tan \theta / c$ ( $c$ - speed of light) is applied to muon arrival times in order to eliminate the distorsions due to the shower inclination. However, there are often experimental difficulties to reconstruct this time-zero with sufficient accuracy. Hence alternatively local arrival times are considered which refer to the arrival time of the first muon, registered locally. In event-by-event observations the individual EAS relative arrival time distributions (global and local) can be characterised by mean values $\Delta \tau_{\text {mean }}$ and by various quantiles like the median $\Delta \tau_{0.5}$, the first and the third quartiles $\Delta \tau_{0.25}$ and $\Delta \tau_{0.75}$, which describe different features of the single distributions. The variation of the distributions of these quantiles, in particular of their mean values and the variances, with the distance $R_{\mu}$ from the shower centre, we call the EAS time profile. In the case of global time parameters these describe the curvature of the shower disk and the shower thickness, while local quantities characterise only the structure of the shower disk. The following discussion is mainly focused on the implications of observations of local time quantities and is based on Monte Carlo simulations of the EAS development, using the code CORSIKA [23] with the hadronic interaction model QGSJET [24] as generator.

\section{Fluctuations of the arrival time of the first muon}

The question to which extent the first detected particle represents the arrival time of the EAS front has been discussed by Villiers et. al. [25].

Following these considerations it can be argued that the arrival time $\Delta \tau_{1}$ of the first muon, relative to a fictitious zero time, representing the muon front, approximated by a sample with a large number $n$ of muons, its expectation value and fluctuations depend on the particular value of the multiplicity $n$. Fig. 2 shows muon arrival time distributions accumulated from many showers with the expectation values $\left\langle\Delta \tau_{1}(n)\right\rangle$ of the arrival time of the first muon for subsamples of different multiplicities.

With increasing $n$ and in the case of infinite time resolution ( $\sigma=0 \mathrm{~ns}$ ) of the timing detector $\left\langle\Delta \tau_{1}(n)\right\rangle$ approaches the fictitious arrival time of the shower front (which appears for small $R_{\mu}$ and infinite time resolution near $\tau_{c}$ ). In addition to the fluctuations of the arrival times of the first muon due to the registered multiplicity (which in practical experimental cases involves also the 


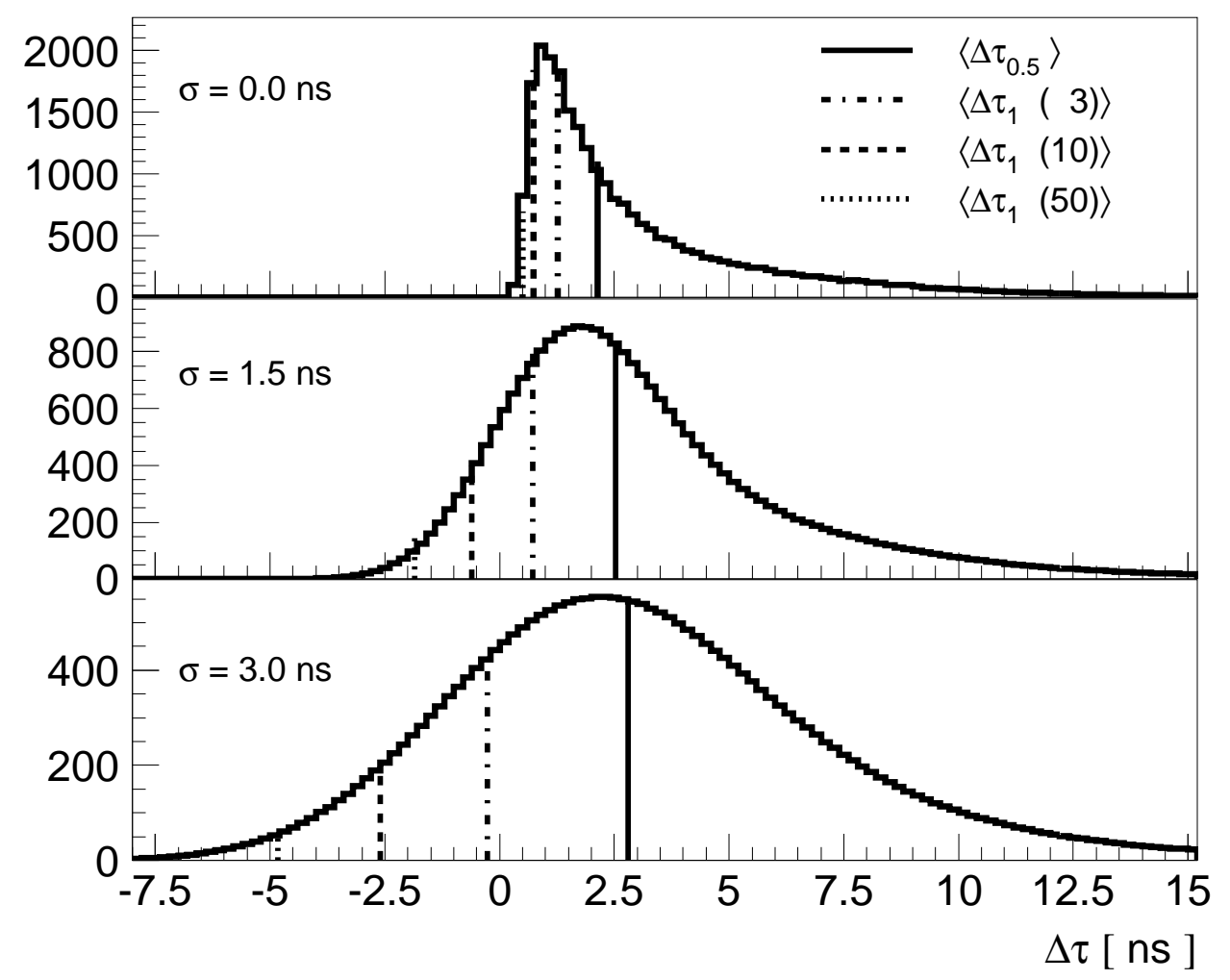

Fig. 2. Muon arrival time distributions $\tau_{1}(n)-\tau_{c}$ with the multiplicity dependence of $\left\langle\Delta \tau_{1}(n)\right\rangle$ and the influence of the time resolution. The shown distributions stem from the accumulation of 100 simulated Fe and 100 simulated proton induced EAS (zenith angle of incidence $0^{\circ}$ ) of $3 \cdot 10^{15} \mathrm{eV}$, observed in the range $70 \mathrm{~m} \leq R_{\mu}<80 \mathrm{~m}$. In the present case of relatively small observation distances from the shower axis differences between Fe and proton induced showers would appear completely covered by the discussed fluctuations.

influence of the detector response) there is the influence of the finite time resolution. It broadens the observed distributions and smears out the original asymmetry. As an example also the expectation for the (global) median $\left\langle\Delta \tau_{0.5}\right\rangle$ is shown. The averaged global median does not depend much on the time resolution. In contrast the local quantities underly the trends of $\left\langle\Delta \tau_{1}(n)\right\rangle$, i.e. they increase with the multiplicity and with the time resolution.

These features are illustrated in Fig. 3 and lead to local shower profiles which are distinctly influenced by the detector qualities (time resolution and response for the muon multiplicity i.e. the detector area) as well as by the natural shower fluctuations. The influence is very pronounced in the central region of the shower with the largest multiplicities. As examples Fig. 4 displays local 


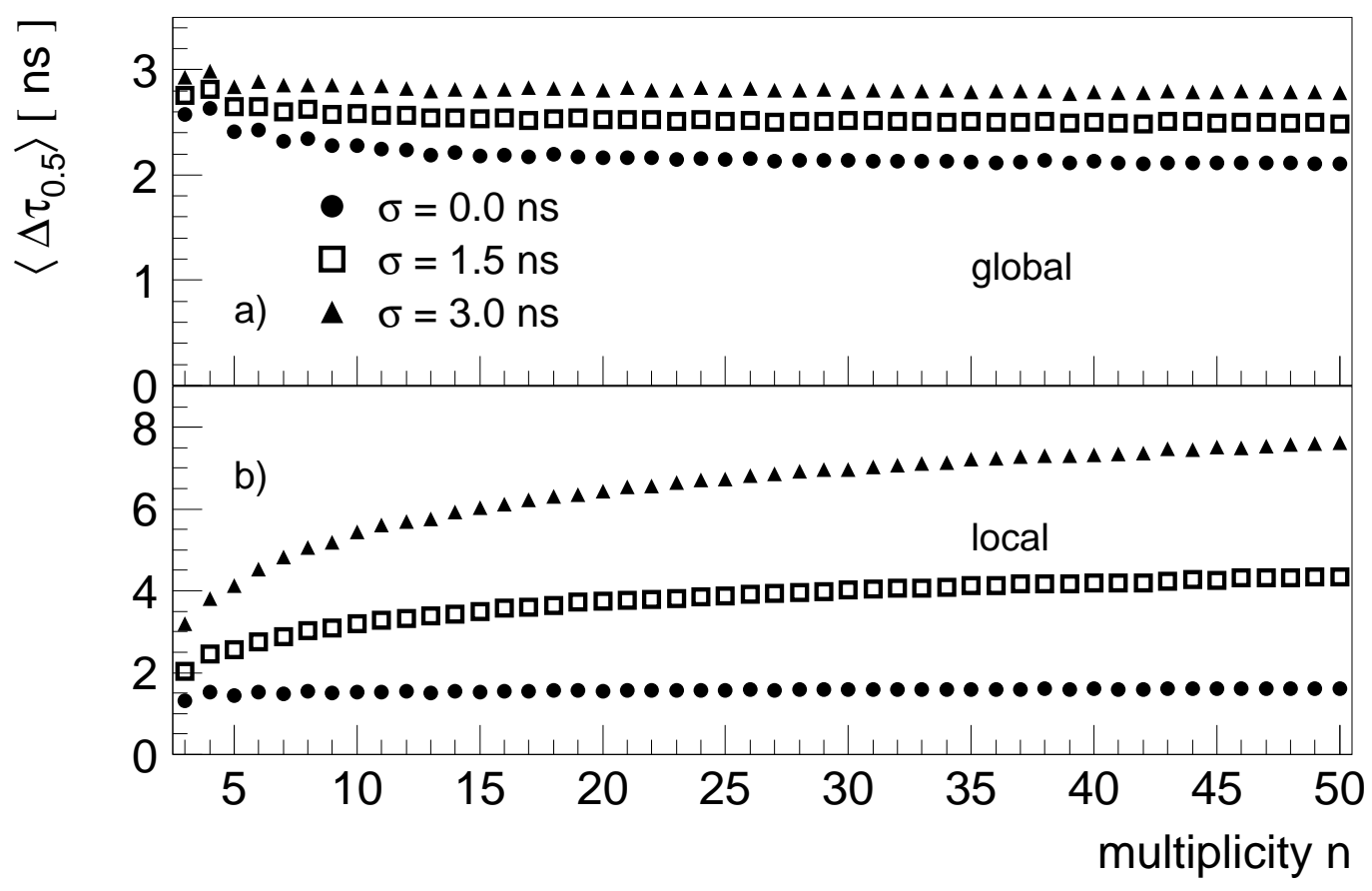

Fig. 3. Multiplicity and time resolution dependence for the mean values of global and local time parameters of single muon arrival time distributions.

time profiles $\left\langle\Delta \tau_{0.5}\right\rangle$ for proton induced showers observed with different time resolution. The profiles approach the ideal case at larger distances from the core (> approx. $250 \mathrm{~m}$ ), where the time resolution loses obviously the influence. It should be noted that local time profiles do exhibit nearly insignificant differences for different masses of the EAS primaries. This feature is contrast to observations of global time parameters [15].

3 How to account for the dependence on the registered muon multiplicity

In actual experimental observations of muon arrival time distributions like in the KASCADE experiment the profiles are derived from EAS events, including all different multiplicities $\left(n>n_{s}\right)$, as registered by the timing detectors. Due to the lateral distribution of the EAS muon component the average (registered) multiplicities in an EAS are depending on $R_{\mu}$. They depend also on the type and energy of the primary, and even if the energy is approximately 


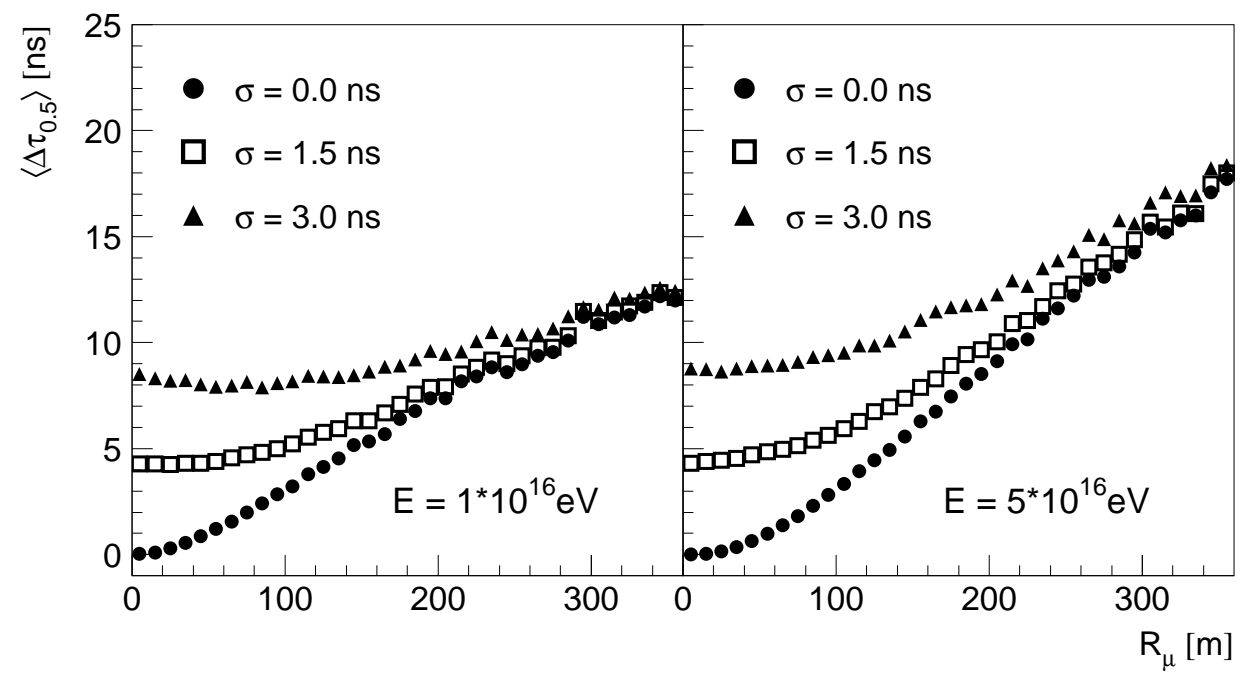

Fig. 4. Local time profiles of proton induced EAS for different primary energies and different time resolution, virtually registered with a detector eye of 400 detectors of $0.5 \mathrm{~m}^{2}$ (approximately like the timing facility of the KASCADE central detector $[12])$.

specified, the observed time profiles originate from a superposition of various multiplicities, varying with the distance from the shower core. That feature leads to interference effects, distorting the predicted quasi-parabolic shape of the time profile. Fig. 5, which displays measured EAS time profiles [26], exhibits this effect.

In principle, it would be desirable to extract the shower profile for each multiplicity separately. However, this attempt would meet serious problems of the statistical accuracy of the results. In addition such a procedure would lead to a less transparent selection of shower events. The main problem, however is that the appearance of the distortions depends on the response qualities of the particular detector arrangement, so that measurements by different detector arrays are hardly directly comparable. There are various ways to approach a representative result about the EAS time structure from measurements of local quantities.

- The standard concept to compare experimental results with theoretical predictions are procedures simulating the experimental conditions and response folded with the predictions and comparing the resulting distributions with the measured results. This will give an impression about agreement or disagreement and on the undistorted time profiles, but does not immediately enable the comparison with other experiments of different (often unknown) quality and conditions. 


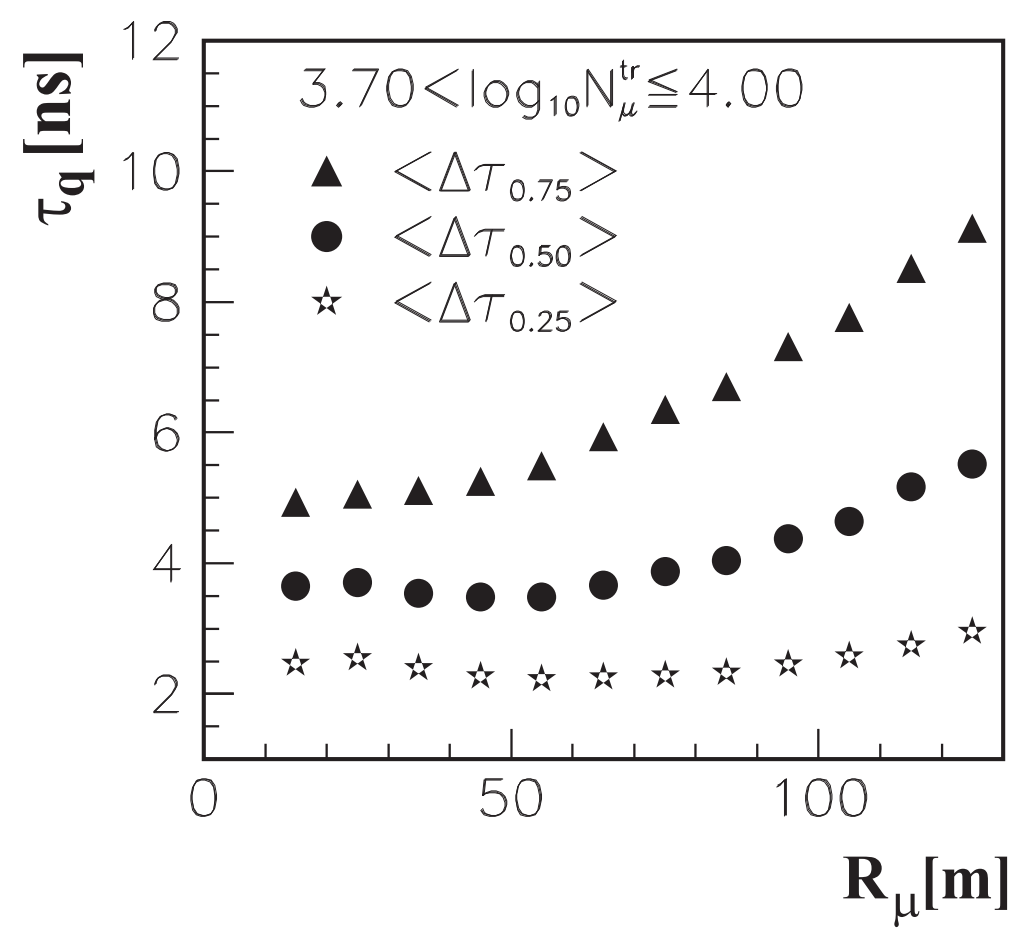

Fig. 5. The observed variation of the mean values of the median, first and third quartile distributions with the distance from the shower axis (from [26]), extracted without multiplicity correction for an EAS sample within a particular range of $\log _{10} N_{\mu}^{t r}$ (indicating [27] the primary energy range of approx. $1.6 \cdot 10^{15} \mathrm{eV}$ to $3.2 \cdot 10^{15} \mathrm{eV}$ ).

- The measured time parameters, deduced for each event from the single muon arrival time distributions registered with varying multiplicity, get scaled to a chosen reference value of the multiplicity by a correction according to predictions by simulation calculations (indicated for the case of the mean value $\Delta \tau_{\text {mean }}$ by a calibration curve in Fig. 3). The appearance of time profiles depends on the choice of the reference multiplicity. The correction procedure needs detailed simulation calculations of muon arrival time distributions of the kind shown in Fig. 3. The procedure has been successfully applied in recent KASCADE experiments $[17,19]$.

- The observation that for global time quantities the influences of the multiplicity and the time resolution are less pronounced, suggests to relate the muon arrival times to the arrival time of the shower centre by simulating the time difference between the arrival time $\tau_{1}$ of the local first muon and the arrival $\tau_{c}$ of the EAS core. In this way [20] the local quantities are transformed into pseudo-global time parameters, which display the EAS time structure rather realistically, but invoke EAS simulations, specified in detail (see also ref. [11]). While only the shape of the arrival time distributions enters in the above multiplicity calibration procedure, the transformation to pseudo-global quantities stresses also the absolute time difference $\tau_{1}-\tau_{c}$ between the arrival of the first muon and of the shower core in the plane perpendicular to the axis. 

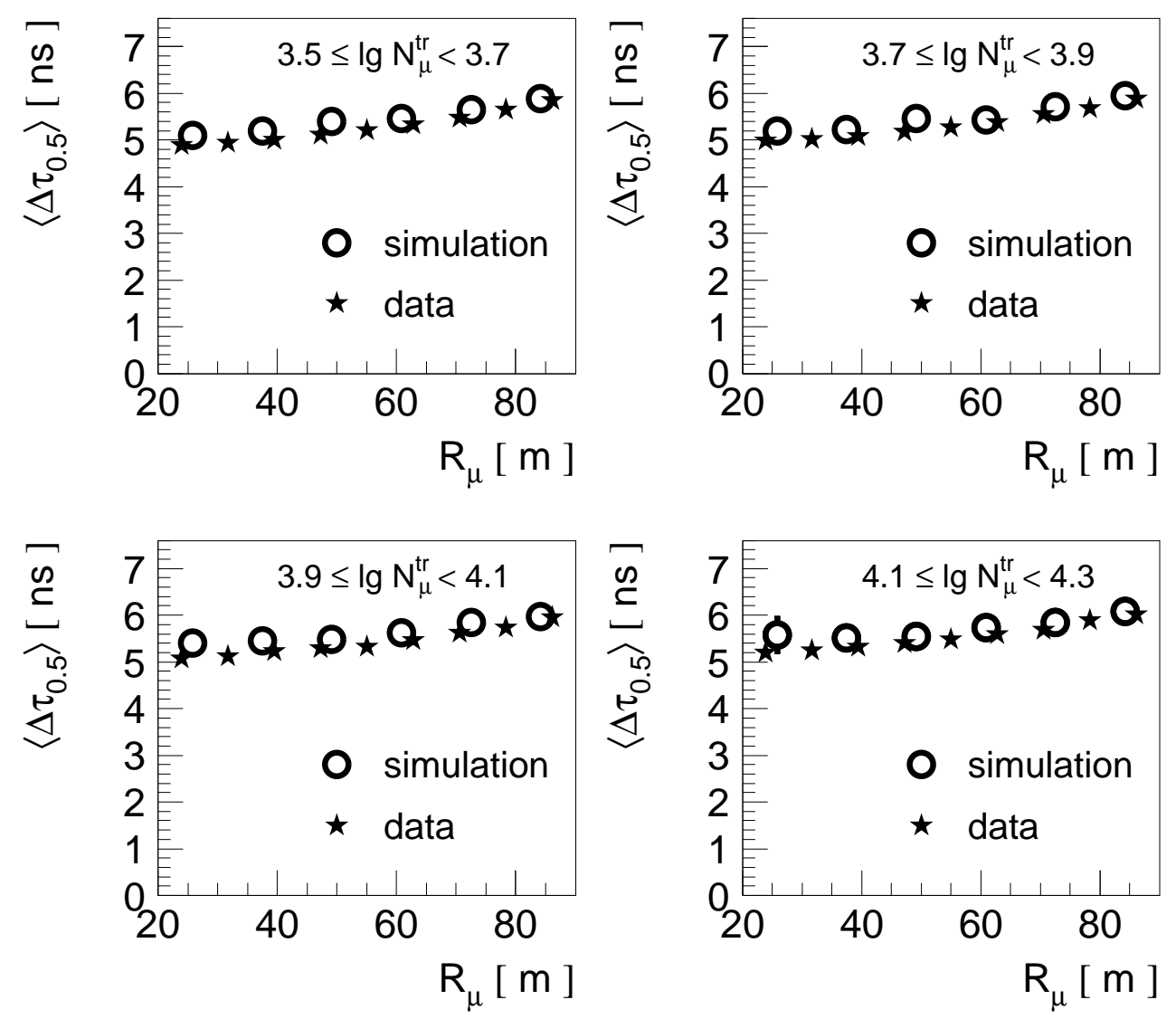

Fig. 6. Local EAS shower profiles $\left(\left\langle\Delta \tau_{0.5}\right\rangle\right)$, corrected for the multiplicity dependence, as compared with predictions of EAS simulations using the Monte Carlo code CORSIKA [23] and taking into account the time resolution, for different EAS muon sizes (corresponding to an energy range from $6 \cdot 10^{14} \mathrm{eV}$ to $6.3 \cdot 10^{15} \mathrm{eV}$ ).

Finally Fig. 6 presents a result of an experimental investigation [19] of EAS time profiles using the KASCADE detector. For different ranges of the truncated muon number $N_{\mu}^{t r}$ (used as approximate energy estimator [27]) and with a consistent correction for the multiplicity dependence, the experimental results are compared with CORSIKA [23] simulations, adopting a mass composition $\mathrm{p}: \mathrm{O}: \mathrm{Fe}=1: 1: 1$.

\section{Concluding remarks}

The shape of measured muon arrival time distributions, which refer to the arrival time $\Delta \tau_{1}$ of the first locally registered muon, experiences some dis- 
tortions which arise from the locally varying superposition of different muon multiplicities (spanning the observed individual event distribution). The effect of multiplicity fluctuations is entangled and amplified in an intricate way with the response and time resolution of the detector setup. Due to the larger intensity of the electromagnetic EAS component and the smaller fluctuations of the local arrival time of the first charged particle, the effects are less pronounced in practical cases of studies of the time structure of the charged particle component [12]. In principle, however, the dependence of $\left\langle\Delta \tau_{1}\right\rangle$ from the observed multiplicity is also present in simulation studies with an ideal time resolution, though mostly covered by the assumption of large collection areas of the ideal detectors. There are various procedures, which remedy the distortions and reveal the basic experimental results of the structure of the EAS disk for a reasonable comparison with theoretical predictions or other experiments. The described procedures are weakly dependent on the used interaction model, governing the EAS development, and on the adopted mass composition. They invoke explicitely EAS Monte Carlo simulations. The described distortions of the observed muon arrival time distributions lose their significance with increasing distance $R_{\mu}$ from the shower axis ( $>250 \mathrm{~m}$ ) and with increasing energy. Nevertheless local time distributions (referring to the arrival time of the first local muon) turn out to be rather insensitive to the mass of primary cosmic rays. This is in contrast of global arrival times [12-15] which refer e.g. to the arrival time of the shower core and measure additionally the curvature of the shower disk.

\section{Acknowledgements}

The consideration are borne out from the experimental studies of the EAS temporal structure by the KASCADE experiment. We acknowledge in particular the clarifying discussions with Dr. Andreas Haungs and Dr. Markus Roth. Two of us (A.F.B. and I.M.B.) would like to thank for the personal support by the WTZ project (RUM 99-005) of the scientific-technical cooperation understanding between Germany and Romania.

\section{References}

[1] P. Bassi, G. Clark and B. Rossi, Phys. Rev. 92 (1953) 441.

[2] J. Linsley, L. Scarsi and B. Rossi, Phys. Rev. Lett. 6 (1961) 485.

[ 3] J. Linsley and L. Scarsi, Phys. Rev. 128 (1962) 2384.

[4] R. Thielert and L. Wiedecke, Z. Phys. 179 (1964) 199.

[ 5] R. Walker and A.A. Watson, Journ. Phys. G: Nucl. Part. Phys. 7 
(1981) 1297.

[6] R. Walker and A.A. Watson, Journ. Phys. G: Nucl. Part. Phys. 8 (1982) 1131.

[ 7] A.A. Watson and J.G. Wilson, Journ. Phys. A: Math. Gen. 7 (1974) 1199.

[ 8] H.E. Dixon and K.E. Turver, Proc. Roy. Soc. London A339 (1974) 171.

[ 9] G. Agnetta et al., Astroparticle Physics 6 (1997) 301.

[10] M. Ambrosio, C. Aramo, L. Colesanti, T.V. Danilova and A.D. Erlykin, Astroparticle Physics 7 (1997) 329.

[11] M. Ambrosio, C. Aramo, L. Colesanti and A.D. Erlykin, Astroparticle Physics 11 (1999) 437.

[12] H.O. Klages et al. - KASCADE Collaboration, Nucl. Phys. (Proc. Suppl.) 52B (1997) 92; Proc. 25th ICRC (Durban, South Africa), Vol. 8 (1997) 297.

[13] H. Rebel, G. Völker, M. Föller and A.A. Chilingarian, Journ. Phys.G: Nucl. Part. Phys. 21 (1995) 451.

[14] T.V. Danilova, D. Dumora, A.D. Erlykin and J. Procureur, Journ. Phys. G: Nucl. Part. Phys. 20 (1995) 961.

[15] I.M. Brancus, B. Vulpescu, H. Rebel, M. Duma, A.A. Chilingarian, Astroparticle Physics 7 (1997) 343.

[16] H. Rebel, in: The Cosmic Ray Mass Composition, Proc. XV Cracow Summer School of Cosmology, 15-19 July, 1996, Lodz, Poland, Uniwersytetu Lodzkiego, ed. W. Tkaczyk (1997) 91.

[17] T. Antoni et al.- KASCADE collaboration, Astroparticle Physics 15 (2001) 149.

[18] W. Hafemann, FZKA-Report 6470, Forschungszentrum Karlsruhe (2000).

[19] R. Haeusler, FZKA-Report 6520, Forschungszentrum Karlsruhe (2000).

[20] A.F. Badea, FZKA-Report 6579, Forschungszentrum Karlsruhe (2001).

[21] J. Engler et al., Nucl. Inst. Meth. A427 (1999) 528.

[22] H. Bozdog et al., Nucl. Inst. Meth. A465 (2001) 455.

[23] D. Heck, J. Knapp, J.N. Capdevielle, G. Schatz and T. Thouw, FZKA-Report 6019, Forschungszentrum Karlsruhe (1998).

[24] N.N. Kalmykov, S.S. Ostapchenko and A.I. Pavlov, Nucl. Phys. 52 B (1997) 17.

[25] E.J. de Villiers, D.J. van der Walt, P.K.F. Grieder and G. van Urk, Journ. Phys. G: Nucl. Phys. 12 (1986) 547.

[26] I.M. Brancus et al.- KASCADE Collaboration, FZKA-Report 6151, Forschungszentrum Karlsruhe (1998).

[27] J.H. Weber et al.- KASCADE Collaboration, 25th ICRC (Durban, South Africa), Vol. 6 (1997) 153. 